\title{
Caractérisation chez les nématodes Meloidogyne Goeldi (Tylenchida) de types virulents vis-à-vis du gène Mi de la tomate dans deux zones maraîchères au Sénégal
}

\author{
F. Berthou ${ }^{1}$, A. Ba-Diallo 2 , L. de Maeyer ${ }^{2}$ et G. de Guiran ${ }^{3}$ \\ avec la collaboration technique de N. Bruguier et M. Dieng \\ 1 Antenne ORSTOM d'Antibes, B.P. 2078, 06602 Antibes, France;
2 ISRA / CDH, B.P. 3120 , Dakar, Sénégal;
3 INRA, station de recherches sur les nématodes, B.P. 2078, 06602 Antibes, France
}

(reçu le 31 janvier 1989; accepté le 5 juillet 1989)

Résumé - Les nématodes à galles du genre Meloidogyne ont été étudiés dans 2 zones maraîchères du Sénégal : les dépressions côtières humides (Niayes) et les périmètres irrigués de l'intérieur, en vue d'analyser l'impact de la diffusion des nouvelles variétés de tomates possédant le gène de résistance $\mathrm{Mi}$ :

- sur la répartition des espèces,

- sur l'apparition ou la dissémination des biotypes virulents vis-à-vis de Mi. Les espèces ont été déterminées par électrophorèse des iso-estérases sur gel d'amidon, la virulence est révélée par l'aptitude au développement sur variété résistante Rossol à $23^{\circ} \mathrm{C}$.

Au total, 4 espèces ont été détectées dans les sondages portant à la fois sur plantes sensibles et tomate résistante : $M$. javanica et $M$. incognita se rencontrent simultanément dans 20 des 24 des localités prospectées. $M$. arenaria est trouvée dans 5 des 24 localités. Une souche nouvelle, désignée par l'abréviation : vs, pour "very slow", eu égard à la vitesse de migration de ses iso-estérases en électrophorèse, est rencontrée dans les Niayes ou à leur contact, ainsi que dans les pépinières du Nord, au total dans 13 des 24 localités.

L'une ou l'autre de ces 4 espèces se développe au champ sur les variétés de tomates résistantes. Dans 2 localités, cela est dû à la suppression de la résistance liée à la hausse de la température, le test négatif de développement sur variété Rossol démontrant l'absence de biotype virulent à $23^{\circ} \mathrm{C}$. Dans d'autres localités, c'est la virulence des peuplements qui est en cause : ces lignées virulentes s'observent dans les 4 espèces. Chez $M$. javanica et $M$. incognita, les proportions de souches virulentes représentent respectivement le tiers et plus de la moitié des cas étudiés. Les souches vs se sont montrées virulentes dans toutes les situations analysées. $M$. arenaria se révèle virulente dans la localité où elle est l'espèce dominante.

Les infestations de variétés résistantes sont avant tout liées à la richesse locale des peuplements du nématode en espèces et biotypes. C'est donc cette diversité qui caractérise les foyers de contamination. Au Sénégal, ces foyers sont localisés dans les Niayes, à leur contact ou dans les pépinières anciennes. La dissémination des souches virulentes doit être évitée, sous peine de compromettre le progrès que représentent, dans la lutte contre Meloidogyne, la création au Sénégal et l'emploi des nouvelles variétés possédant le gène Mi.

Meloidogyne - virulence - tomate - gène Mi - foyers de contamination - électrophorèse - lutte culturale

Summary - Characterization of virulent (Mi gene resistance breaking) biotypes of root-knot nematodes Meloidogyne Goeldi (Tylenchida) in two vegetable growing areas of Senegal. Species and virulent (resistancebreaking) biotypes of root-knot nematodes (Meloidogyne spp.) were studied in 2 major vegetable growing areas of Senegal to assess the risk of using tomato cultivars carrying the Mi gene for resistance.

Species were identified by starch gel electrophoresis of isoesterases and virulence biotypes revealed by their ability to reproduce on the resistant tomato cultivar Rossol at $23^{\circ}$.

Mixed populations of M. javanica and M. incognita were present in 20 and M. arenaria in 5 localities of the 24 localities sampled. In addition, an undescribed species characterized by its esterase profile was found in 13 of these localities.

In 2 localities surveyed, Mi gene resistance break was related to the weakening of this resistance due to the high soil temperatures. In the others, populations were found to contain virulent biotypes. Those of $\mathrm{M}$. javanica and 
M. incognita occurred, respectively, in one third and one half of the fields sampled, whereas all populations of the new species were virulent. In the locality where M. arenaria was the dominant root-knot species, it proved to be virulent.

The infestation of resistant cultivars was directly related to the diversity in species and biotypes of local nematode populations. Based on these results, it is essential that infestation of virulent biotypes of root-knot species be contained when using resistant tomatoes in control strategies. The dissemination of virulent populations must be avoided.

Meloidogyne - virulence - tomato - Mi gene - host range - electrophoresis - farming control

\section{INTRODUCTION}

Les nématodes à galles (Meloidogyne spp.) représentent un facteur limitant de la production en cultures maraîchères, particulièrement dans les régions tropicales. Au Sénégal, où ces productions représentent un potentiel économique important, c'est le cas notamment de la tomate, cultivée en saison des pluies pour les besoins locaux. Les variétés résistantes, productives dans ces conditions et capables de réduire les populations du nématode, représentent un moyen de lutte de plus en plus utilisé. Les lignées : Xina et Rossol, et les F1, Hope $\mathrm{N}^{\circ} 1 \mathrm{H}$ et Small-Fry, offrent une résistance très marquée, due à la présence dans leur génome du gène Mi. Elles sont fréquemment cultivées.

Mais cette stratégie peut être contrariée, car ces variétés sont parfois elles-mêmes attaquées. Les attaques manifestent la présence de biotypes virulents, c'est-à-dire de souches capables de proliférer sur ces cultivars, de manière d'autant plus intense qu'ils sont plus productifs. Par conséquent, dans les pays tropicaux, la possiblité de contrôler le parasite par l'emploi des variétés de tomates résistantes est controversée (Luc \& Reversat, 1985).

En ce qui concerne le Sénégal, les souches étaient initialement considérées comme n'apparaissant que par pression de sélection à la suite de la culture de variétés résistantes (Netscher, 1970), comme cela avait déjà été observé dans les régions tempérées (Kehr, 1966). Mais, ultérieurement, il a été signalé au Sénégal (Prot, 1984) qu'existaient aussi des souches spontanément virulentes, trouvées dans la région du Fleuve. II est donc important de déterminer chez quelles espèces et avec quelles fréquences apparaissent, dans les peuplements de nématodes de ce pays, de tels biotypes virulents responsables du maintien de la pression parasitaire.

Cela constitue l'objet de cette étude, réalisée dans les 2 régions maraîchères principales : les Niayes, dépressions inondées en saison des pluies, où ces nématodes se rencontrent à la périphérie, et les parties hautes, représentées par les plaines du relief dunaire, saisonnièrement sèches.
Des échantillons ont été prélevés au champ sur diverses cultures et plantes adventices, et les Meloidogyne déterminés au laboratoire par électrophorèse. Une attention particulière a été portée aux Meloidogyne développés sur les tomates résistantes. Une des difficultés réside dans la perte de résistance chez la tomate à température supérieure à $28^{\circ} \mathrm{C}$ (Laterrot \& Pécaut, 1965), ce qui donne une fausse impression de virulence (tout en réduisant la pression de sélection). Des tests de virulence sur variété résistante ont donc parallèlement été pratiqués au laboratoire à température contrôlée.

\section{Matériel et Méthodes}

Au total, 24 localités ont été prospectées pour l'inventaire d'espèces et biotypes (Fig. 1). Dans 15 d'entre elles, les nématodes ont été prélevés à la fois : sur des plantes maraîchères, comprenant des cultivars résistants de tomates, et sur les plantes multiplicatrices, spontanées ou cultivées. Dans 8 autres localités, les tomates résistantes, non installées à l'époque, ne faisaient pas partie des plantes cultivées. Ces localités sont marquées d'un astérisque sur la Figure 1. Dans la localité 4, les plantes échantillonnées consistaient uniquement en tomates résistantes, la flore multiplicatrice, naturelle ou adventice, étant absente sur toute l'étendue du périmètre.

Ces localités sont repérées sur la Figure 1 par des numéros : 1 à 17 ou des lettres majuscules : A à G, les chiffres désignant des localités de l'intérieur du pays (ou région 1), les lettres, des localités de Niayes (ou région II). Le nombre total de sondages réalisés s'élève à 150. Individualisées avec leurs pontes, plusieurs dizaines de femelles sont prélevées lors de chaque sondage, sur la culture et les végétaux environnants. Parmi ces derniers, on trouve, en culture associée : papayer (Carica papaya) et persil (Petroselinum sativum), pratique courante dans les Niayes. Par ailleurs, de nombreuses plantes spontanées sont souvent présentes, dont de nombreuses Composées et Amaranthacées.

Au laboratoire, les femelles sont dégagées des racines et broyées individuellement, soit dans de l'eau sucrée à $20 \%$, soit sur une pastille de papier filtre Whatman ' 1 '. Les pontes sont prélevées préalablement, mises à éclore dans l'eau et éventuellement utilisées en fonction des besoins pour réaliser les tests de virulence. Les broyats de femelles sont analysés en électrophorèse sur supports, soit de polyacrylamide, soit d'amidon. Les espèces sont identifiées selon les profils des iso-estérases, déterminés initialement sur support de polyacrylamide (Dalmasso \& Bergé, 1983). 


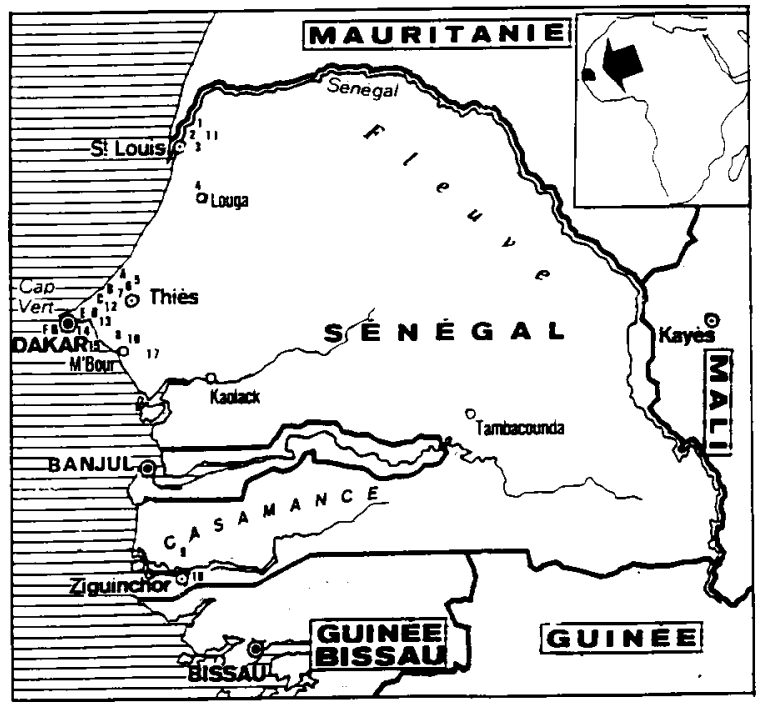

Fig. 1. Localisation de 24 points de prélèvements pour l'inventaire de Meloidogyne spp. en culture maraîchère au Sénégal.

Région I (localités de l'intérieur) : (1)-Savoigne pivot 4 ("); (2)Lampsar pépinière; (3)-N'Diol parcelle K; (4)-Louga pivot SERPA; (5)-Noto Gouye Diama; (6)-M'Bayack maison familiale; (7)-Gorom I; (8)-Gandigal; (9)-Coubalan ( $\left.{ }^{*}\right)$; (10)-Diabir ( $\left.{ }^{*}\right)$; (11)-Savoigne pépinière (*); (12)-Sangalkam; (13)-C.D.H. Cambérène; (14)-Rufisque Keur Daouda; (15)-Rufisque 'tuyau'; (16)-Nianing ("); (17)-Sandiara;

Région II (les Niayes) : (A)-M'Boro; (B)-Retba; (C)-Niaga; (D)Pikine Nord (*); (E)-Pikine Sud; (F)-Thiaroye ("); (G)-Hann (").

Le gel d'amidon est utilisé en routine dans cette étude comme support d'électrophorèse : bien que les résultats fussent moins lisibles, ce procédé nous a paru préférable pour gagner en rapidité d'exécution, tout en évitant la manipulation de produits toxiques. Le gel est élaboré de la façon suivante : $10 \mathrm{~g}$ d'amidon hydrolysé SIGMA et $1 \mathrm{~g}$ de polyvinylpyrolidone insoluble sont mis en suspension dans $100 \mathrm{ml}$ de tampon tris-citrate $\mathrm{pH}: 8$ contenant $1 \mathrm{~g}$ de tris-(hydroxyméthyl) aminométhane. La suspension est chauffée à $80^{\circ} \mathrm{C}$, puis coulée dans le moule de dimensions (23,0 x 8,0 $x$ $0,3) \mathrm{cm}$. La migration est effectuée sous $200 \mathrm{~V}$, les électrodes étant plongées dans un tampon : borate de sodium $\mathrm{pH}: 8$ contenant $18,5 \mathrm{~g}$ d'acide borique.

Dans le test de virulence au laboratoire, chaque ponte éclose, comportant 100 larves L2 environ, est placée dans un godet type "Nalgène" de $5 \mathrm{ml}$ contenant du sol stérile, sur lequel on a complanté, 4 j après les semis (en boîte de Petri), 2 plantules, l'une résistante, de la variété Rossol, et l'autre multiplicatrice, de la variété-hôte Casaque Rouge. Cette dernière est aisément reconnaissable par son feuillage. Après $10 \mathrm{j}$, les 2 plantules provenant d'un même godet sont repiquées ensemble dans un tube de $40 \mathrm{ml}$ en polyéthylène, contenant du sol stérile, et conservées, durant 7 semaines, au laboratoire, éclairé et climatisé à $23^{\circ} \mathrm{C}$.

A l'issue d'un cycle de reproduction du nématode, les pontes formées sont comptées:

- la souche issue d'un individu identifié est comptée pour avirulente : $(-)$, si les racines du plant de la variété Rossol sont indemnes, alors que celles du plant de la variété Casaque Rouge, infesté par la même ponte, extériorisent de 4 à 50 pontes;
- elle est au contraire notée comme virulente : (+), si Rossol est elle-même attaquée, au même degré que Casaque Rouge.

Les cas rares d'attaques discrètes (moins de 4 pontes) ne sont pas pris en considération dans cette étude.

\section{RÉSULTATS}

\section{Caractérisation des espèces}

Les 4 profils iso-estérasiques rencontrés et utilisés pour l'identification des espèces se distinguent aisément, soit en gel de polyacrylamide, soit en gel d'amidon : la Figure 2 confronte les profils spécifiques réalisés à partir des mêmes souches et obtenus suivant les 2 procédés. Des profils d'iso-estérases les plus lentes aux profils d'iso-estérases les plus rapides, nous observons:

\section{Le premier profil}

De bandes à migration lente, il caractérise les lignées désignées vs., abréviation pour very slow (Esbenshade \& Triantaphyllou, 1985).

\section{Le second profil}

En position moyenne, il caractérise l'espèce $M$. incognita, désignée en abréviation in.; à propos de vs. et in., notons que les profils obtenus sont identiques, en gel de polyacrylamide et en gel d'amidon.

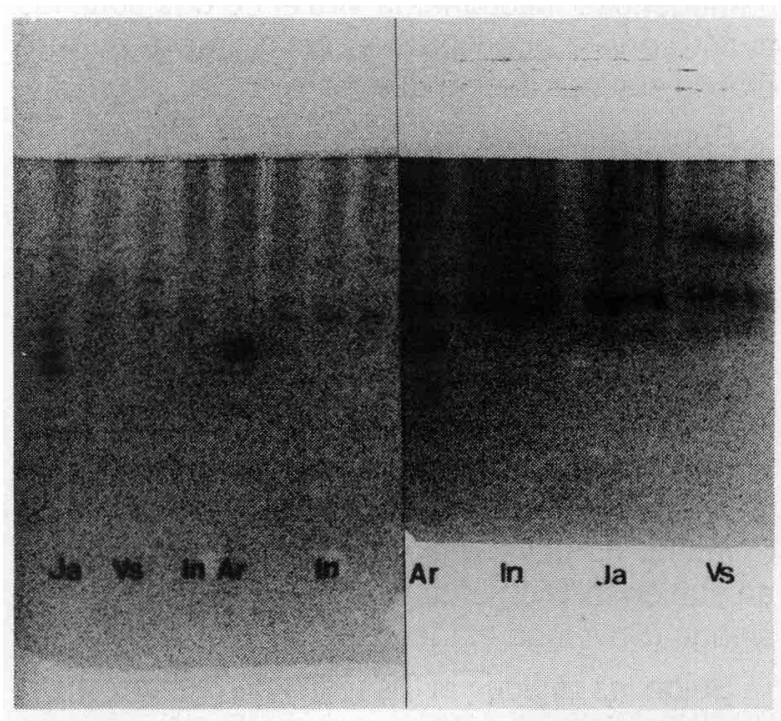

Fig. 2. Profils d'iso-estérases des 4 espèces de Meloidogyne identifiées en gel de polyacrylamide (à gauche) et en gel d'amidon (à droite) (Ja : M. javanica, In: $M$. incognita, Vs: lignées vs., Ar. : M. arenaria) 


\section{Le troisième profil}

De bandes en positions mixte, moyenne et distale, il caractérise l'espèce $M$. javanica, abréviation : ja. Les 3 bandes obtenues en gel de polyacrylamide se réduisent à 2 en gel d'amidon. Cette différence ne traduit pas de modification du profil isoenzymatique. II s'agit d'artefact, la qualité de résolution étant seule en cause.

\section{Le quatrième profil}

De bandes à migration rapide, il caractérise l'espèce $M$. arenaria, abréviation : ar. Mais les deux profils, sur gel d'amidon et sur gel de polyacrylamide, sont distincts dans ce cas : respectivement, 3 bandes ( 1 moyenne et 2 rapides) contre 2 bandes rapides (pour toutes les souches analysées provenant du Sénégal). Réelle, quoique inexpliquée, cette différence ne met pas en cause la possibilité de reconnaître les espèces les unes des autres, par l'un ou l'autre de ces procédés.

\section{Caractérisation des peuplements}

Dans chaque espèce ou lignées (vs.), et pour un total de 150 prélèvements, les Tableaux I et II présentent :

- l'effectif d'individus prélevés sur diverses plantes hôtes.

- le nombre des souches trouvées virulentes: $(+)$ et avirulentes : (-) vis-à-vis de Mi par le test de virulence en laboratoire, test ayant été pratiqué au total 401 fois.

En effet, les espèces se reproduisant par parthénogenèse mitotique, la virulence des souches ayant servi à l'identification spécifique a pu être établie sur leur descendance.

Pour l'ensemble du Sénégal, $M$. javanica et $M$. incognita se rencontrent simultanément dans 20 des 24 localités, et $M$. arenaria est présent dans 5 localités seulement. Par rapport au nombre total de prélèvements $(N=150), M$. javanica est retrouvée 55 fois $(37 \%), M$. incognita : 52 fois $(35 \%), M$. arenaria : 8 fois $(5 \%)$. Les lignées vs. se rencontrent 35 fois (23\%), dans 13 localités : elles sont présentes dans les Niayes ou à leur contact, mais on ne les retrouve, dans l'intérieur du pays, que dans la région du Fleuve, en station (N'Diol) et dans 2 pépinières à son voisinage (Savoigne et Lampsar).

Selon les régions et les types de plantes, en \% par rapport aux totaux des prélèvements par région (région I: $\mathrm{N}=89$ et région $\mathrm{II}: \mathrm{N}=61$; plantes multiplicatrices : $\mathrm{N}=122$ et plantes présumées résistantes : $\mathrm{N}=28$ ), la composition spéci- fique des peuplements est peu variable : $M$. arenaria, $5-7 \%$; lignées vs., $21-26 \%$; $M$. incognita, $31-37 \%$; et $M$. javanica, $36-40 \%$. En particulier, la variation de la composition spécifique entre plantes multiplicatrices et tomates résistantes est faible : la plus élevée concerne M. incognita $(32-37 \%)$; la variation concernant les lignées vs. étant : $21-25 \%$.

Pour chaque espèce ou lignée vs. rencontrées, l'analyse permet de préciser en outre la proportion des sites pour lesquels on a trouvé au moins 1 individu virulent : les biotypes virulents comptent pour 1 s'ils sont présents au moins une fois, et leur nombre est rapporté au nombre de sites pour lesquels les tests sont effectués $(\mathrm{N}=49)$. Ainsi, les proportions d'individus virulents, dans l'ordre ja, in, vs, ar, sont respectivement : pour la totalité des échantillons : $5 / 16$, $12 / 19,12 / 12$ et $1 / 2$, soit, dans le sous-échantillon des plantes multiplicatrices : $2 / 11,11 / 18,9 / 9$ et $1 / 2$, et dans celui des tomates résistantes : $3 / 5$, $1 / 1,3 / 3$ et 0 .

A noter que sur les 9 localités où les peuplements Meloidogyne ont pu être examinés sur tomates résistantes, on trouve 2 cas de peuplements avirulents, à savoir : 2 peuplements comportant exclusivement, ou surtout : $M$. javanica. (Louga, M'Bayack).

\section{DISCUSSION-CONCLUSION}

La présence de nématodes phytoparasites du genre Meloidogyne sur la plupart des plantes maraîchères en sols sableux au Sénégal oblige les producteurs à aménager la succession des cultures de manière à limiter leur prolifération en deçà du seuil où ils créent des dommages. Les plantes non-hôtes préconisées dans ce but au Sénégal, comme l'arachide : Arachis hypogaea (Netscher, 1975), le fonio : Digitaria exilis (Sarr \& Prot, 1985), l'herbe de Guinée : Panicum maximum (de Guiran \& Germani, 1980), et les variétés résistantes de tomate : Lycopersicon esculentum, ont un effet bénéfique directement visible sur la culture suivante : mais, de cette liste, seules les dernières sont couramment employées comme moyen de lutte contre Meloidogyne, ce qui nous a permis d'évaluer leur efficacité, lors de la saison chaude, en rapport avec la diversité de la composition des peuplements de nématodes parasites de plantes dans les zones maraîchères prospectées.

En effet, dans le cas des peuplements de nématodes comportant 1 ou 2 espèces seulement (Louga, M'Bayack), nous avons observé, au champ, l'attaque localisée de la variété résistante Rossol par M. javanica sans qu'apparais- 
Tableau I. Composition des peuplements par espèces ( $M$. javanica (ja.), incognita (in.), arenaria (ar.) et lignées vs,(vs) ) en nombre d'individus totaux, et en nombre d'individus virulents : +, et avirulents : - (entre parenthèses) pour 17 localités de la région I (l'intérieur du pays).

\begin{tabular}{|c|c|c|c|c|c|c|c|}
\hline & \multirow[t]{2}{*}{$\begin{array}{c}\text { hôtes } \\
\text { spontanés * }\end{array}$} & \multicolumn{5}{|c|}{$\begin{array}{l}\text { hôtes } \\
\text { cultivés ** }\end{array}$} & \multirow[t]{2}{*}{$\begin{array}{c}\text { totaux } \\
\text { individus }\end{array}$} \\
\hline & & 1 & II & III & IV & $V$ & \\
\hline 1 & & & $\begin{array}{r}34 j a . \\
4 i n .\end{array}$ & & 106ja. (22-) & & $\begin{array}{l}\text { 140ja. }(22-) \\
\text { 4in. }\end{array}$ \\
\hline 2 & & & $\begin{array}{c}40 v s . \\
2 j a .\end{array}$ & & & $20 v s$. & $\begin{array}{l}60 v s . \\
2 j a .\end{array}$ \\
\hline 3 & & & $15 v s$. & & $\begin{array}{l}9 v s .(3+) \\
27 j a .\end{array}$ & 40vs. & $\begin{array}{l}64 v s . \\
27 j a .\end{array}$ \\
\hline 4 & & & & & & 32ja. (7-) & 32ja. (7-) \\
\hline 5 & & & & $\begin{array}{l}19 j a . \\
10 i n .\end{array}$ & & $\begin{array}{l}24 j a .(2+) \\
\text { 5in. }\end{array}$ & $\begin{array}{l}\text { 43ja. }(2+) \\
15 \text { in. }\end{array}$ \\
\hline 6 & & & $\begin{array}{l}23 j a . \\
15 i n\end{array}$ & 20ja. (8-) & & 20ja. (16-) & $\begin{array}{l}63 \mathrm{ja} .(24-) \\
15 \mathrm{in} .\end{array}$ \\
\hline 7 & & & & $10 \mathrm{in}$ & & 40in. $(3+, 3-)$ & 50 in $(3+, 3-)$ \\
\hline 8 & & & & 10ja. & $\begin{array}{l}44 j a . \\
14 i n .\end{array}$ & $10 j a$. & $\begin{array}{l}64 j a . \\
14 i n\end{array}$ \\
\hline 9 & & $\begin{array}{c}1 \text { ja. }(1+) \\
13 i n .(11-)\end{array}$ & $\begin{array}{l}113 j a .(14+, 2-) \\
9 \mathrm{in}\end{array}$ & $\begin{array}{l}27 j a .(8+, 8-) \\
2 \text { in. }\end{array}$ & $\begin{array}{r}29 j a . \\
4 i n .\end{array}$ & & $\begin{array}{l}\text { 170ja. }(23+, 10-) \\
28 \text { in. }(11-)\end{array}$ \\
\hline 10 & 30ja. (16-) & $\begin{array}{l}3 j a . \\
56 \mathrm{in} .\end{array}$ & $\begin{array}{l}49 j a . \\
\text { 6in. }\end{array}$ & & $\begin{array}{l}20 j a . \\
26 i n .\end{array}$ & & $\begin{array}{l}\text { 102ja. (16-) } \\
\text { 88in. }\end{array}$ \\
\hline 11 & & & $\begin{array}{l}47 \text { vs. }(13+) \\
\text { 7in. }(3+, 1-\text {, } \\
6 j a .\end{array}$ & $\begin{array}{c}12 v s . \\
4 i n . \\
3 j a .\end{array}$ & & & $\begin{array}{l}59 \text { vs. }(13+) \\
11 \text { in. }(3+, 1-) \\
\text { 9ja. }\end{array}$ \\
\hline 12 & & $\begin{array}{c}12 \text { vs. }(6+) \\
8 \text { in. (8-) }\end{array}$ & & & $\begin{array}{l}19 v s . \\
13 i n .(1-) \\
1 \text { ja. }\end{array}$ & $5 v s$. & $\begin{array}{l}36 \text { vs. }(6+) \\
21 \text { in. }(9-) \\
\text { ija. }\end{array}$ \\
\hline 13 & $\begin{array}{l}25 v s . \\
4 i n .\end{array}$ & & $\begin{array}{l}60 v s .(7+) \\
\operatorname{gin} .(2+, 5-)\end{array}$ & & $45 v s .(10+)$ & 15vs. $(15+)$ & $\begin{array}{c}145 \text { vs. }(32+) \\
13 \text { in. }(2+, 5-)\end{array}$ \\
\hline 14 & & $20 j a$. & $7 j a$. & $\begin{array}{l}6 j a . \\
4 i n .\end{array}$ & $\begin{array}{l}13 j a . \\
10 j a .\end{array}$ & $10 j a$. & $\begin{array}{l}30 j a . \\
36 j a . \\
4 i n .\end{array}$ \\
\hline 15 & & & & 32ja. (2-) & & $\begin{array}{l}20 v s . \\
4 j a . \\
35 v s .(4+)\end{array}$ & $\begin{array}{l}20 \mathrm{vs} . \\
36 \mathrm{ja.}(2-) \\
35 \mathrm{vs} .(4+)\end{array}$ \\
\hline & & & & $\begin{array}{c}\text { 9in. (2-) } \\
\text { 29ar. (10-) }\end{array}$ & & tin. & $\begin{array}{l}\text { 10in. (2-) } \\
29 \text { ar. (10-) }\end{array}$ \\
\hline 16 & & & $\begin{array}{l}19 \mathrm{in} . \\
11 \mathrm{ja} .\end{array}$ & $7 a r$. & & $\begin{array}{c}92 a r .(15+, 1-) \\
\text { 6in. }(1+, 2-) \\
17 j a .(1-)\end{array}$ & $\begin{array}{l}99 a r .(15+, 1-) \\
25 \mathrm{in} .(1+, 2-) \\
28 \mathrm{ja} .(1-)\end{array}$ \\
\hline 17 & & & & & $\begin{array}{l}26 \text { in. }(14+) \\
\text { 4ar. } \\
4 j a .\end{array}$ & $\begin{array}{r}14 i n \\
\text { Gar. }\end{array}$ & $\begin{array}{l}\text { 40in. }(14+) \\
10 a r . \\
4 j a .\end{array}$ \\
\hline
\end{tabular}

" hôtes spontanés : Amaranthus hybridus (10), Emilia sonchifolia (13); "* hôtes cultivés (selon les différentes localités); I : Lactuca sativa, cv. Pierre Bénite (9, 10 et 12); Petroselinum sativum (14); II: Solanum melongena, cv. Black Beauty (1, 10, 13 et 16); Solanum aethiopicum: cv. Soxna (3), Dianki (9), et divers (13); Capsicum chinense, cv. Safi (2 et 11); Solanum tuberosum, cv. Premiere (6); III : Abelmoschus esculentus, cv Puso (5, 6, 7, 8, 9 et 15); Brassica oleracea, cv. Marché de Copenhague (11, 15 et 16); Daucus carota, cv. Nantaise (14). IV : tomates variétés-hótes, cv. Roma (1, 3, 8, 9, 10, 14 et 17$)$ et $X$ in $(12,13)$; $V$ : tomates réputées résistantes, $c v$. Xina $(2,3,5,6,7,12,13$ et 14), Rossol (4), Small-Fry (8 et 17$)$ et Hope $n^{\circ} 1 \mathrm{H}(15$ et 16$)$.

sent d'individus virulents dans le test en laboratoire, à 2 reprises au sein de chacun de ces peuplements : l'attaque serait attribuable à l'élévation localisée de la température, qui estompe l'action du gène Mi. Nous avons observé en outre que la contamination du sol était plus massive sur ces emplacements, en liaison, d'une part, à Louga, avec le précédent cultural : pomme de terre, particulièrement infestée précédemment à l'emplacement considéré, et, d'autre part, à M'Bayack, avec la présence de culture associée de gombo : Abelmoschus esculentus dans la partie du 
Tableau II.Composition des peuplements par espèces ( $M$. javanica (ja.), incognita (in.), arenaria (ar.) et lignées vs (vs.)) en nombre d'individus totaux, et en nombre d'individus virulents : + , et avirulents : - (entre parenthèses) pour 7 localités de la région II. (les Niayes).

\begin{tabular}{|c|c|c|c|c|c|c|c|}
\hline & \multicolumn{2}{|c|}{$\begin{array}{c}\text { hôtes } \\
\text { spontanés * }\end{array}$} & \multicolumn{4}{|c|}{ hôtes cultivés ** } & \multirow[t]{2}{*}{$\begin{array}{c}\text { totaux } \\
\text { individus }\end{array}$} \\
\hline & 1 & 2 & 1 & 11 & III & $I V$ & \\
\hline A & $18 \mathrm{in}$ & & $\begin{array}{l}7 v s .(7+) \\
3 \text { ja. }\end{array}$ & & $\begin{array}{l}35 \mathrm{in} .(4-) \\
12 \mathrm{ja} .\end{array}$ & $\begin{array}{l}\text { 19ja. }(4+, 10-) \\
10 \mathrm{vs} . \\
\text { i in. }\end{array}$ & $\begin{array}{l}54 \mathrm{in} .(4-) \\
34 \mathrm{ja} .(4+, 10-) \\
17 \text { vs. (7-) }\end{array}$ \\
\hline B & & $\begin{array}{c}38 j a . \\
32 i n . \\
2 v s .\end{array}$ & $\begin{array}{r}31 \mathrm{in} . \\
19 j a . \\
2 v s .\end{array}$ & $\begin{array}{l}44 \text { in. }(4+, 9-) \\
35 j a . \\
8 v s .\end{array}$ & $\begin{array}{c}28 v s . \\
2 j a .\end{array}$ & & $\begin{array}{l}107 \text { in. }(4+, 9-) \\
94 j a . \\
40 \mathrm{vs.}\end{array}$ \\
\hline C & $18 \mathrm{in}$ & & $\begin{array}{l}\text { 43in. (4-) } \\
\text { 3ar. }\end{array}$ & $\begin{array}{l}224 \text { in. }(13-) \\
20 \text { ja. }\end{array}$ & & $\begin{array}{l}25 \mathrm{ja} .(3+, 1-) \\
20 \mathrm{in} . \\
5 \mathrm{ar} .\end{array}$ & $\begin{array}{l}305 \mathrm{in} .(17-) \\
45 j a .(3+, 1-) \\
8 \text { ar. }\end{array}$ \\
\hline D & & & $\begin{array}{l}18 v s . \\
9 j a .(2-) \\
5 \text { in. (5-) }\end{array}$ & & $20 v s .(6+)$ & & $\begin{array}{c}\text { 38vs. (6+) } \\
\text { 9ja. (2-) } \\
\text { 5in. (5-) }\end{array}$ \\
\hline$E$ & & & $\begin{array}{l}11 \text { in. (5-) } \\
9 v s .\end{array}$ & $\begin{array}{l}160 j a . \\
40 i n .\end{array}$ & $\begin{array}{l}9 j a . \\
\text { 6in. }\end{array}$ & $\begin{array}{l}48 v s .(5+) \\
10 \mathrm{in.} \\
1 \mathrm{ja} .\end{array}$ & $\begin{array}{l}\text { 170ja. } \\
67 \text { in. }(5-) \\
57 \text { vs. }(5+)\end{array}$ \\
\hline$F$ & & & $\begin{array}{l}40 \mathrm{in} .(8+, 3-) \\
10 \mathrm{vs} .\end{array}$ & $\begin{array}{l}45 \text { vs. }(4+) \\
18 \text { in. } \\
\text { 1ja. (1-) }\end{array}$ & $\begin{array}{r}20 i n . \\
1 j a .\end{array}$ & & $\begin{array}{l}78 \mathrm{in} .(8+, 3-) \\
55 \mathrm{vs} .(4+) \\
2 \mathrm{ja} .(1-)\end{array}$ \\
\hline $\mathrm{G}$ & & & $\begin{array}{l}71 \text { in. }(5+, 17-) \\
6 j a . \\
5 v s .(2+) \\
7 \text { ar. }\end{array}$ & $\begin{array}{l}58 \text { vs. } \\
60 \mathrm{in} .(3+, 7-) \\
33 \mathrm{ja} .\end{array}$ & $\begin{array}{c}114 \text { vs. }(14+) \\
\text { 16in. }(3+, 7-) \\
\text { 1ja. }(1-)\end{array}$ & & $\begin{array}{l}\text { 177vs. }(16+) \\
\text { 147in. }(11+, 31-) \\
\text { 40ja. }(1-) \\
\text { 7ar. }\end{array}$ \\
\hline
\end{tabular}

- hôtes spontanés - selon les différentes localités : 1 : Alternanthera nodiflora (C); Amaranthus hybridus (A); 2 : Crassocephalum sambamense et Lau. naea taraxacifolia (B); "* hótes cultivés - selon les différentes localités : I : Lactuca sativa, cv. Pierre Bénite (B, D, E, F et G); Abelmoschus esculentus, cv. Puso (F); Brassica oleracea, cv. Marché de Copenhague (A, B, C et F); Ipomoea batatas cv. Ndargu (B); Manihot esculenta (B); II : Carica papaya et Petroselinum sativum (B, C, E et $G)$; Solanum aethiopicum (F); III : tomates variétés-hôtes, $c v$. Roma $(B)$ et $X$ in $(A, C$ et $E) ; I V:$ tomates réputées résistantes, cv. Xina (A et C) et Small-Fry (E).

champ attaquée. Dans ce type de situation, les variétés résistantes ont constitué néanmoins un moyen de lutte culturale efficace contre les nématodes à galles.

II est courant d'objecter la précarité de ce procédé de lutte, du fait de la possibilité, pour la plupart des espèces Meloidogyne spp., de briser la résistance contrôlée par le gène Mi. Mais, pour plusieurs raisons, dans ces régions où les peuplements manifestent une variabilité réduite, cette éventualité ne devrait pas entraver la diffusion des variétés résistantes. En effet, parmi les facteurs qui tempèrent la pression exercée par le gène $\mathrm{Mi}$ sur le nématode, signalons l'action de plantes annexes ou adventices qui représentent autant de niches épargnant la pression exercée par la culture aux nématodes qu'elles hébergent (Kehr A.E., 1966). Par ailleurs, la plupart des espèces de ce genre, et notamment celles que nous avons étudiées ici, se reproduisent par 
parthénogenèse mitotique, ce qui restreint les potentialités évolutives de ce nématode (Dalmasso, 1980). A cette restriction s'ajoute la réduction du nombre de générations annuelles du nématode en zone sub-sahélienne, en relation avec la quiescence anhydrobiotique des pontes (Demeure, 1978). Ces divers aspects de la biologie du nématode à galies laissent penser que, dans ces conditions particulières, la pression du gène $\mathrm{Mi}$ sur les populations ne joue qu'un rôle restreint dans l'apparition des types virulents.

A l'inverse, dans les peuplements riches en espèces (Cambérène, Thiaroye et Rufisque), les biotypes virulents sont mis en évidence dans plus d'une espèce et surtout parmi les espèces les plus abondantes : soit chez $M$. incognita et lignées vs. dans les deux premiers, soit chez $M$. arenaria et $M$. incognita dans le dernier. Ces peuplements plus polymorphes se situent dans les Niayes ou à leur contact, c'est-à-dire là où l'on rencontre les zones les plus favorables à l'expansion des Meloidogyne, et là aussi où les variétés résistantes de tomate de saison des pluies sont le plus fortement attaquées. Dans ce cas, les plantes arbustives annexes (Taylor et al. 1978, Prot, 1986), constituent, comme les plantes adventices étudiées ici, de nombreux réservoirs natureis d'inoculum qui réalisent un système de réinfestation continu (Luc \& Reversat, 1985). Cet effet apparaît dans la constance de la composition spécifique des peuplements selon les régions et les plantes-hôtes considérées.

Le Sénégal constitue donc un véritable champ d'essai pour évaluer l'impact des variétés résistantes comme moyen de lutte culturale contre le nématode à galles, en zone sub-sahélienne et aussi dans les zones plus humides de Niayes, qui sont des zones-refuges pour la flore (Adam, 1953 ) et pour la faune associée. En fait, dans les 2 régions, de Niayes ou de l'intérieur du pays, la variabilité des populations par localité est plus forte que ne le laissait penser l'étude antérieure (Netscher, 1970), tant pour le pourcentage de populations virulentes, toutes espèces confondues, que pour le nombre d'espèces, déterminé antérieurement en fonction de l'ornementation cuticulaire de la région périnéale. Elle est beaucoup plus forte que dans les régions tempérées, où l'on a estimé le pourcentage de populations virulentes à $1-3 \%$ (Dalmasso \& Missonnier, 1986) : au Sénégal, cette proportion s'élève à $80 \%$, surtout en liaison avec la présence de lignées vs.. Cette variabilité est dans une large mesure indépendante de la culture des variétés résistantes; elle est plus importante dans les Niayes ou à leur contact que dans l'intérieur du pays. L'on peut admettre ainsi que au Sénégal, les souches virulentes vis-à-vis du gène Mi sont en majorité spontanées, leur présence paraissant antérieure à la diffusion des variétés résistantes.

Si leur existence est plus ancienne, et si leur zone de conservation actuelle se situe dans les Niayes, la dissémination des souches virulentes au dehors est un phénomène certainement récent, comme l'atteste, dans le Nord, la présence de la lignée $v s$. dans les pépinières, mais pas dans les champs. Il est donc important de ne pas propager ces souches indésirables dans les régions où elles ne sont pas naturellement présentes. La désinfection des substrats de pépinières est particulièrement recommandée, cellesci étant très souvent installées sur des sols sableux. En outre, il est préférable d'installer la pépinière à proximité immédiate de l'emplacement réservé à la culture. On notera que cette pratique est de règle sur les grands pivots d'irrigation du Nord-Sénégal.

Dans les Niayes, et dans les 2 périmètres maraîchers de la Petite Côte (Rufisque et Nianing), où coexistent de nombreux types virulents, la diffusion des variétés résistantes risque d'être peu efficace et de favoriser l'extension de ces types indésirables. Par ailleurs, il arrive que les variétés résistantes soient moins fertiles et donnent des fruits de calibre inférieur (Laterrot, 1971). Pour cette raison, en terrain non infesté au Sénégal, la variété-hôte Xin est souvent préférée à la variété résistante Xina. Ainsi, la diversité des situations est telle qu'il convient de réserver l'emploi des variétés de tomates résistantes aux zones où la pression de sélection sur les populations de nématodes a quelque chance de s'exercer. Les situations les plus propices à l'emploi des variétés résistantes sont celles où, en fonction de nos critères, les peuplements de nématode à galles manifestent la variabilité locale la plus restreinte.

Les lignées $v s$ analysées sont toutes virulentes vis-à-vis du gène $\mathrm{Mi}$ de résistance chez la tomate, comme cela s'observe ailleurs à propos de $M$. hapla (espèce qui n'est pas représentée au Sénégal). Une lignée virulente provenant de N'Diol, la souche numérotée 14568 par Prot, a été assimilée, en fonction de l'ornementation de la région périnéale, à $M$. arenaria (Prot, 1984), puis à $M$. incognita (Sarr \& Prot, 1985, et Fargette, 1988), ce qui marque, en ce cas, l'imprécision du critère morphologique de détermination. Mais le nouveau critère biochimique iso-estérase établit sans conteste qu'il s'agit là, très précisément, d'un zymodème particulier, vs., qui est largement représenté au Sénégal aussi bien sur les rives du lac Retba, dans la flore spontanée (Crassocephalum sambamense) et le papayer, que dans 
presque toutes les Niayes aménagées par l'homme, autour de Hann et de Pikine, et dans des pépinières du Nord-Sénégal. Outre sa fréquence de virulents vis-à-vis de Mi, sans équivalence au Sénégal, une autre caractéristique est à signaler: sa virulence sur le terrain vis-à-vis de la patate douce : Ipomoea batatas, cv. N'dargu, et du piment : Capsicum chinense, cv. Safi., qui sont résistants à d'autres espèces de Meloidogyne. Dans ce cas, la détermination en électrophorèse d'un zymodème nouveau nous met peut-être sur la voie de la détermination d'une espèce nouvelle, intéressant l'améliorateur.

La recherche de nouvelles sources de résistance chez $C$. chinense reste à faire. Cette investigation est importante dans la mesure où les gènes de résistance du poivron ( $C$. annuum) ne sont pas des gènes homologues de Mi (Hendy et al., 1983). On peut espérer élargir la base de sélection des variétés résistantes à partir du matériel végétal local. En effet, la présence de divers types virulents dans ces zones a permis peut-être le tri progressif de variétés locales dotées de résistance par la pression parasitaire, si elle s'exerce sur les populations depuis suffisamment longtemps.

Pour poursuivre l'étude de la virulence du parasite, il serait donc intéressant de développer l'exploitation de ce laboratoire naturel que constituent les zones maraîchères du Sénégal, en testant notamment en conditions locales les sources génétiques de la résistance aux nématodes à galles d'autres Solanacées, soit d'origine locale, soit aussi d'origine sauvage.

\section{RÉFÉRENCES BIBLIOGRAPHIQUES}

Adam J. (1953) Note sur la végétation des Niayes de la presqu'île du Cap Vert (Dakar-A.O.F.). Bull.Soc. Bot. Fr. 153-158

Dalmasso A. (1980) Le nématode Meloidogyne et la tomate de conserve. Pépiniéristes Hortic. MaraichersRev. Hortic. 205, 29-32

Dalmasso A. \& Bergé J.B. (1983) Enzyme polymorphism and the concept of parthenogenetic species, exemplified by Meloidogyne. In: Concepts in Nematode Systematics (A.R. Stone, H.M. PLatt, L.F. Khalil eds) Academic Press, London, pp 187-196

Dalmasso A. \& Missonnier J. (1986) La lutte intégrée contre les nématodes des cultures : intérêt des variétés résistantes. Phytoma. Déf. Cult. 378, 13-16

Demeure Y. (1978) Les causes de la survie de certains nématodes phytoparasites (Scutellonema cave- nessi et Meloidogyne spp.) pendant la saison sèche dans le sahel sénégalais. Thèse 792, Univ. Claude Bernard, Lyon

Esbenshade P.R. \& Triantaphyllou A.C. (1985) Use of enzyme phenotypes for identification of Meloidogyne species. J. Nematol. 17, 6-20

Fargette $M$. (1988) Présence de races $B$ du nématode phytoparasite Meloidogyne incognita en Côte d'Ivoire et leur caractérisation par l'électrophorèse des estérases. C.R. Acad. Sci. Paris 306, Sér. III, 437-440

de Guiran G. \& Germani G. (1980) Fluctuation des populations de Meloidogyne incognita dans un sol cultivé en climat tropical humide. Rev. Nématol. 3, 51-60

Hendy H., Pochard E. \& Dalmasso A. (1983) Identification de deux nouvelles sources de résistance aux nématodes du genre Meloidogyne chez le piment, Capsicum annuum L. C.R. Acad. Agric. Fr. 817-822

Kehr A.E. (1966) Current status and opportunities for the control of nematodes by plant breeding, USDA Agricultural Research Service, 30-110, 126-138

Laterrot H. (1971) Méthodes culturales et variétés résistantes. In: Les Nématodes des Cultures. ACTA Paris, p. 573

Laterrot H. \& Pécaut P. (1965) Effect of high temperature on the resistance of Tomato variety "Anahu" to Meloidogyne incognita. Rep. Tomato. Gen. Coop. 15, 38-39

Luc M. \& Reversat G. (1985) Possibilités et limites des solutions génétiques aux affections provoquées par les nématodes sur les cultures tropicales. C.R. Acad. Agric. Fr. 781-791

Netscher C. (1970) Les nématodes parasites des cultures maraîchères au Sénégal. Cah. ORSTOM, sér. Biol. 11, 207-229

Netscher C. (1975) Studies on the resistance of groundnut to Meloidogyne spp. in Senegal. Cah. ORSTOM, Sér. Biol. 10, 227-232

Prot J.C. (1984) A naturally occuring resistance breaking biotype of Meloidogyne arenaria on tomato. Reproduction and pathogenicity on tomato cultivars Roma and Rossol. Rev. Nématol. 7, 23-28

Prot J.C. (1986) Sensibilité de sept légumineuses arborescentes aux nématodes Meloidogyne javanica, $M$. incognita, Scutellonema cavenessi et Dolichorhynchus elegans. Rev. Nématol., 9, 416-418

Sarr E. \& Prot J.C. (1985) Pénétration et développement de juvéniles d'une souche de Meloidogyne javanica et d'une race $\mathrm{B}$ de $M$. incognita dans la racine de fonio (Digitaria exilis Stapf). Rev. Nématol. 8, 59-65

Taylor D.P., Netscher C. \& Germani G. (1978) Adansonia digitata (Baobab), a newly discovered host for Meloidogyne spp. and Rotylenchulus reniformis : agricultural implications. PI. Dis. Rept. 62, 276-277

Triantaphyllou A.C. (1979) Cytogenetics of root-knot nematodes. In: Root-knot Nematodes (Meloidogyne species); Systematics, Biology and Control (F. Lamberti, C.E. Taylor eds) Academic Press, Londres, pp 85-109 